\title{
The Theory of Essence. II. An Electromagnetic-Quantum Mechanical Model of Interactionism
}

\author{
J. Kenneth Arnette, Ph.D. \\ Colorado State University
}

\begin{abstract}
The theory of essence, based on the physics of electromagnetism and quantum mechanics, solves the problem of interaction resulting from Descartes's dualistic conception of human nature. The theory is empirically based in phenomena consistently reported by near-death experiencers. These phenomena are divided into four categories of interaction experienced by the dissociated mind (or essence), each of which is analogous to properties of electric dipoles. I compare the theory of essence with Descartes's interactionist propositions and propose an experimental test of the theory.
\end{abstract}

Contemplation of the relationship between mind and body is a time-honored human activity. Such musings occurred in antiquity, as evidenced by Homer's Odyssey (Popper and Eccles, 1977), and debate continues today (Dennett, 1991). In the history of this debate, René Descartes is a pivotal figure. Karl Popper (Popper and Eccles, 1977) asserted that before Descartes, all significant philosophers (of whom we know enough to comment) were either explicitly or implicitly dualistic interactionists, except perhaps for the Greek thinkers Leucippus and Democritus, a view espoused a century ago by Friedrich Lange (1865/1925).

J. Kenneth Arnette, Ph.D., received a doctorate in Physical Chemistry from Florida State University and is currently a Ph.D. candidate in Counseling Psychology at Colorado State University. The author is indebted to Mr. Doug Woody and Professor Scott Hamilton for valuable discussions and for their reviews of the manuscript. Reprint requests should be addressed to Dr. Arnette at the Department of Psychology, Colorado State University, Fort Collins, CO 80526. 
Descartes's Meditations on First Philosophy (1641/1986) contained his conception of what became known as interactionism, the doctrine that mind and body were two different things that interacted with each other. Meditations spurred strong reactions from other philosophers of the time. Both Pierre Gassendi and Thomas Hobbes were driven to respond to Descartes with materialistic philosophies (Sarasohn, 1985) and the works of Baruch Spinoza, Gottfried Leibniz, and Nicholas de Malebranche were partly motivated by what was seen as a central weakness in Descartes's theory: the question of how mind and body, being very different in nature, could interact (Radner, 1985).

It is useful to summarize the interactionist propositions Descartes put forth in his Meditations. (1) A "substance" is defined as "a thing capable of existing independently" (Descartes, 1641/1986, p. 30). (2) Mind and body both are substances, but (3) mind is thinking and non-extended, whereas (4) body is non-thinking and extended:

On the one hand I have a clear and distinct idea of myself, in so far as I am simply a thinking, non-extended thing; and on the other hand I have a distinct idea of body, in so far as this is simply an extended, non-thinking thing. And accordingly, it is certain that I am really distinct from my body, and can exist without it. (Descartes, $1641 / 1986$, p. 54)

(5) An extended substance is defined as three-dimensional, with shape and motion:

I distinctly imagine the extension of the quantity (or rather of the thing which is quantified) in length, breadth, and depth. I also enumerate various parts of the thing, and to these parts I assign various sizes, shapes, positions and local motions; and to these motions I assign various durations. (Descartes, $1641 / 1986$, p. 44)

(6) The body, as an extended substance, also has the property of exclusionary occupation of space: "By body I understand whatever has a determinable shape and a definable location and can occupy space in such a way as to exclude any other body..." (Descartes, 1641/1986, p. 17). (7) Mind and body differ further in that the former is indivisible and the latter divisible: "The body is by its very nature always divisible, while the mind is utterly indivisible" (Descartes, 1641/1986, p. 59). (8) The human being is an interactive union of mind and body: "For these sensations of hunger, thirst, pain and so on are nothing but confused modes of thinking which arise from the union and, as it were, intermingling of the mind with the body," and "My body, 
or rather my whole self, in so far as I am a combination of mind and body, can be affected by the various beneficial or harmful bodies which surround it" (Descartes, 1641/1986, p. 56).

Daisie Radner (1971) has investigated this last point. She reviewed the writings of Descartes, including his letters to Princess Elizabeth of Bohemia, in order to establish his views on the question of union. Radner found that Descartes de-emphasized his doctrine of the complete disparity between mind and body, saying that mind and body formed a "substantial union" that allowed causal interaction. Descartes proposed that there were four types of "primary notions": thought (or mind), extension (or body), those things common to both thought and extension (such as appetites, emotions, sensations, existence, unity, and duration), and the union of mind and body. Thus, he conceived it possible that mind and body could share some characteristics, although his stated common features do not give insight into the interaction mechanism. He further explained that while mind and body are substances in the Cartesian sense, the human being is a composite of the two and incomplete without both.

Thus, there are eight propositions of Descartes's interactionism, but no answer to the obvious question, Pierre Gassendi's objection: how does the interaction work? More specifically, what is the nature of the mind/body union, and how can this union be causal? Although some have claimed that no such problem exists (Loeb, 1981), and although Descartes himself denied the importance of such questions (Radner, 1971, 1985), it is quite clear that a problem of interaction does exist (Radner, 1985) and that dualistic interactionism as a theory is incomplete in the absence of a model for interaction. The aim of this paper is to propose such a model.

Ideally, a theory of interactionism should be grounded in empirical data. It is difficult, however, to identify reliable empirical evidence that unequivocally supports the dualistic viewpoint. There are phenomena, such as out-of-body experiences, past-lives regressions, neardeath experiences, and psychic experiences, that can be interpreted from a dualistic perspective. While each of these phenomena is controversial and subject to multiple explanations, the near-death experience does offer a reproducible body of data that is difficult to explain from a completely materialistic (materialist monist) view of human nature.

In a recent paper reviewing results from research on the neardeath experience (NDE) within the context of the mind/body problem (Arnette, 1992), I found that the NDE provides strong support for 
dualism. Furthermore, I suggested that marked similarities exist between the observations of NDErs and predictions made by the general theory of relativity (Einstein, 1961). In particular, the tunnel phenomenon (Moody, 1975; Ring, 1980, 1984; Sabom, 1982) often reported by NDErs bears close resemblance to the relativistically predicted wormhole (Misner, Thorne, and Wheeler, 1973), which in principle can connect our universe with other universes. The tunnel and other features of the NDE are consistent with a conception of interdimensional travel provided by general relativity theory.

On the basis of the scientific foundation formed by the similarities between the NDE and general relativity, I proposed that the human being is composed of two parts: body and essence (Arnette, 1992). The essence is that part of the person that survives the death of the body. It is composed of something other than matter, and thus is not subject to many of the physical laws of this universe, such as gravity or other constraints on motion. The essence contains the essential elements of the human personality or consciousness. The theory of essence holds that upon bodily death, the essence is disengaged from both the body and this universe (or spacetime), and travels via a connecting wormhole to locales imperceptible to us while in physical form. The theory suggests that the observations made by the essence during NDEs constitute empirical data, and that these data could be used to construct a scientifically valid model of human nature based on well-established principles of physics and chemistry.

The present paper represents the first step in this model construction. From NDE data concerning various types of interactions that the essence experiences, a model of those interactions is built upon a foundation formed by the theories of electromagnetism and quantum mechanics. From the model, a structural definition of mind is proposed and then compared with Descartes's interactionist propositions.

\section{Method}

NDE data take the form of interviews, with transcriptional material being analyzed with a qualitative research methodology (Tesch, 1990). Interview and other textual data can be analyzed for their content, in order to identify regularities (or irregularities) in the data, categories represented, and relationships among such categories. A method for accomplishing these tasks is content analysis, which has been broadly defined as "any technique for making inferences by ob- 
jectively and systematically identifying specified characteristics of messages" (Holsti, 1969, p.14). Content analysis can be performed for a variety of purposes. Those relevant to NDE data analysis include describing the characteristics of communications, auditing communication contents against standards, and identifying trends or categories in communication content (Holsti, 1969).

Symbolic interactionism (Blumer, 1969) is a school of thought with methods of analysis and theory construction that build upon content analysis. The methodology of this school aims at "the formulation of propositions about relationships among categories of data, which the researcher weaves into a 'theoretical scheme' " (Tesch, 1990, p. 83). This method generally consists of three steps (Becker and Geer, 1982): (1) the observations are used to construct hypotheses about the existence of a phenomenon and its possible relationships to other phenomena; (2) the generality of the phenomena and their possible relationships is determined; and (3) the individual findings are used to verify, reject, or modify the propositions.

These steps are not necessarily sequential or distinct, but rather occur interactively throughout the analysis. The researcher also looks for exceptions to the phenomena discovered; finding none establishes the generality of the phenomena. At the end of the process, the researcher has extracted meaningful, general categories from the data and is in a position to make logical connections between categories, that is, to build a theory based on the data.

The tools of qualitative research have been applied to NDE data in major studies by Raymond Moody (1975), Kenneth Ring (1980, 1984), and Michael Sabom (1982). Moody's study (1975) was the first systematic investigation of the near-death experience. The impetus for his work was the strong similarity of features among various NDE reports he had encountered. Moody's sample consisted of 150 NDE cases. His analysis drew from this sample the general categories that constitute the NDE.

Ring (1980) replicated and extended Moody's work by identifying and content analyzing a sample of 102 NDE cases, and verified the generality of the phenomena Moody had found. Ring then proposed a model of the NDE that included five stages representing increasing depth of the experience. Sabom (1982) collected a sample of 116 cases and performed content analysis on the resulting data. He confirmed the results and categories of Moody and Ring. Sabom classified NDEs as autoscopic (viewing one's own body), transcendental (entering a 
different reality), and composite (having autoscopic and transcendental elements).

Sabom's study is especially noteworthy. Not only did he provide important verification of the work of the two previous researchers, but he also offered a unique perspective for near-death research from his position as a cardiologist. Sabom identified ten cases of autoscopic NDEs occurring in operating rooms, and accessed the medical records for these cases. He compared the NDE interview data with these records-which the patients had never seen-and found that in every case, the NDEr's report was consistent with the medical records. A great many of the observations thus verified could have only been obtained visually by the NDEr. Of course, the NDEr typically offered more detail than appeared in the records, but where the records were explicit, the interview data were consistent. In terms of content analysis, this comparison of records with interviews is an example of auditing communication content against standards (Holsti, 1969).

In one case, for example, the NDEr supplied minute visual details of the behavior of two meters on the defibrillator being used to revive his body. Sabom compared these details with his own knowledge of the defibrillator model used at the time of the incident, and found the NDEr's description to be extremely accurate. Cases such as this one are very difficult to explain from the materialist monist perspective, since the unconscious and dying NDEr must have been able to see the details reported. In the language of Thomas Kuhn (1970), these cases are the anomalies that can induce paradigm change. These anomalies have been the motivation for the theory discussed below.

In this paper, NDE data are approached from the symbolic interactionist perspective. The major studies outlined above yielded a set of phenomena that have been established as general and reproducible by three independent researchers. From these major studies, four classes of interaction are identified: interactions of the essence with the NDEr body, with the NDEr brain, with other bodies, and with other essences. All but the first of these classes represent general observations for which no exceptions were found. The essence/NDEr body interactions reported are themselves exceptions, since the NDEr typically does not remember reentering the body (Moody, 1975). Further comments on this point are made below.

After the identification of interaction classes, the next steps in the process are to look for logical connections between these classes and to construct an explanatory theory from the connections. The unify- 
ing perspective that emerges from the four interactional classes of data is that of electromagnetic-quantum mechanical theory. This perspective organizes the data in a consistent and logical fashion, revealing relationships between seemingly unconnected phenomena. The theory thus constructed allows predictions to be made, one of which is presented below in the Discussion.

\section{Empirical Observations}

For each interactional class discussed below, three examples are cited, drawn from two sources. Within a given category, each account is from a different NDEr. Any emphasis indicated is that of the subject.

\section{Interactions with the Body}

NDEs occurring in hospital operating rooms provide an unusual opportunity to learn about the forces at work between the essence and the NDEr's physical body. Revival of the NDEr's body by electric shock provides a distinct off/on transition for the body; NDErs observing this transition during the autoscopic phase, which Ring (1980) called Stage 2, are in a unique position to make important observations about the essence/body reunion. The first three NDE excerpts were from surgical patients whose hearts had stopped and who had observed their own revivals by defibrillator.

Case 1:

I was up there at the ceiling, watching them work on me. When they put the shocks on my chest, and my body jumped up, I just fell right back down to my body, just like dead weight. The next thing I knew, I was in my body again. (Moody, 1975, p. 82)

\section{Case 2:}

The nurse was on this side of the bed with that machine. She picked up them shocker things and put one there and one right there [pointing to appropriate places on chest] and I seen my body flop like that. . . . It seemed like it just took me and slammed me together, you know. It seemed like I was apart and then like two forces coming together in a crash. It seemed like I was up here [pointing to ceiling] and it grabbed me and my body and forced it back, pushed it back. (Sabom, 1982. p. 35) 
Case 3:

I was sitting up there somewhere and I could look down. . . . They thumped me a second time. . . Then I reentered my body-a transition that was just like that [the snap of a finger]. (Sabom, 1982, p. 35)

Once their physical bodies were resuscitated, each of these NDErs reported an immediate return to the body. In the second account, this returning was likened to the expression of a powerful force, and in the first account, to the force of gravity.

It can be seen from these first three examples that NDErs vary somewhat in the way they describe an apparently common experience. This variation is consistent with the psychological principle of individual differences in attention, concentration, memory, and verbal skills. It is also important to realize that NDEs are difficult to describe in ordinary language. In most respects, the phenomena reported are beyond our common physical experience. Despite these potential sources of variation, the agreement among the reports is strong.

\section{Interactions with Other Bodies}

While there is clearly an interaction between the essence and the NDEr's living body, quite a different situation arises in cases where the essence interacts with other living bodies. These cases again occurred during the autoscopic phase of the NDE.

Case 4:

The doctors and nurses were pounding on my body to try to get IV's started and to get me back. .. . I tried to move their hands to keep them from beating on my body, but nothing would happen. I couldn't get anywhere. It was like-I don't really know what happened, but I couldn't move their hands. It looked like I was touching their hands and I tried to move them-yet when I would give it the stroke, their hands were still there. I don't know whether my hand was going through it, around it, or what. I didn't feel any pressure against their hands when I was trying to move them. (Moody, 1975, p. 44)

Case 5:

As they came by they wouldn't seem to notice me. They would just keep walking with their eyes straight ahead. As they came real close, I would try to turn around, to get out of their way, but they would just walk through me. (Moody, 1975, p. 45) 
Case 6:

I'm trying to stop them [the doctors]. I really did try to grab ahold of them and stop them, because I really felt happy where I was. . . . I actually remember grabbing the doctor. . . . [What happened?] Nothing, absolutely nothing. It was almost like he wasn't there. I grabbed and he wasn't there or either

I just went through him or whatever. (Sabom, 1982, p. 33)

Whether intentional or accidental, physical contact with people is impossible for the essence to achieve. It seems that the essence can occupy the same space as a body, without interacting with it.

\section{Cognitive Effects of Dissociation}

Once the essence has acclimated to its dissociation from the body, it begins to notice some of its attributes. One such attribute that is important for the present purpose is the nature of the essential thinking process. Knowledge of this process will give insight into essence/brain interaction, since one may then make a comparison of cognition in the dissociated and integrated states.

Case 7:

Things that are not possible now, are then. Your mind is so clear. It's so nice. My mind just took everything down and worked everything out for me the first time, without having to go through it more than once. (Moody, 1975, p. 51)

Case 8:

I just thought that I was dying at that particular time. It didn't even affect me as far as being scared. Of course, being up there and knowing your mind was alive, but my mind was very much alive. I could think very clearly, even though I was considered dead [laughs]. (Ring, 1980, p. 89)

Case 9:

[How would you describe how your mind was working while in this state?] Very cognitive. Really, very rational. Very determined. (Ring, 1980, p. 91)

The first account directly states, and the others imply, that cognitive functioning is noticeably improved by essence/body dissociation. Thinking is clearer, more rational, and more efficient when separated from the brain. 


\section{Interactions with Other Essences}

Although the essence cannot interact physically with others, it can indeed interact mentally with others. This is evident when essences encounter each other, as in the transcendental phase (Sabom, 1982) or Stage 3 or 4 of the NDE (Ring, 1980). Also, in some cases when the essence is near people, as in the autoscopic phase or Stage 2, the NDEr reports sensing thoughts.

Case 10:

I could see people all around, and I could understand what they were saying. I didn't hear them, audibly, like I'm hearing you. It was more like knowing what they were thinking, exactly what they were thinking, but only in my mind, not in their actual vocabulary I would catch it the second before they opened their mouths to speak. (Moody, 1975, p. 52)

Case 11:

While I was dead, in this void, I talked to people-and yet, I really couldn't say that I talked to any bodily people. . . . Whenever I wondered what was going on, I would always get a thought back from one of them. . . I always got an answer back for every question that I asked. They didn't leave my mind void. (Moody, 1975, p. 58)

Case 12:

[When you heard the voice, it was a male voice. Did you actually hear the voice, or-?] It was like it was coming into my mind. It was like I didn't have any hearing or sight or anything. It was like it was being projected into my mind. (Ring, 1980, p. 93)

The data indicate that the essence can interact with other essences by exchanging information at a distance in the form of thought. The essence can also receive the thoughts of those in physical form before the words are spoken.

\section{Theoretical Interpretation}

The task at hand is to construct a theory within which the above observations form a consistent whole. The aim is to show that a certain perspective, employing concepts from the physics of electromagnetism and quantum mechanics, unifies the data and demonstrates that each phenomenon is a logical result of the nature of the essence. In pursuing this aim, I will use analogical reasoning: the analogy 
will formally employ the behavior of electric dipoles, but will also informally appeal to the behavior of magnetic dipoles, since magnetism is more common in experience.

\section{Essence / Body Interaction}

There is evidently a great attractive force at work between the essence and the NDEr's body. The action of this force can be seen in the first three quotations cited above. The onset of the force is apparently associated with the resumption of the biological functioning of the body, which in the examples coincides with electric shock administered in the operating room. What is the nature of this essence/body force?

While it is conceivable that the body generates some unknown force that can act upon the essence, it is more reasonable and fruitful to propose that a known force is at work. A strong candidate for this operative force is electromagnetism, since the central nervous system is an electrochemical generator of electromagnetic energy. Neural impulses are, in fact, electromagnetic field pulses of a fixed amplitude and duration (Carlson, 1986). The pulses are transmitted down the neuronal axon by the opening of sodium and then potassium channels in a given region of the axonal membrane, thus turning the signal on and then off. The next segment of axon responds in a like manner, and the process continues until the signal has been relayed to the synapse at the axon's end. Information is transmitted via the frequency and pattern of these pulses. The living body is therefore a producer of a temporally varying and spatially extended electric field.

In order to identify electromagnetism as the operative essence/body force, it must also be established that the essence generates or is associated with an electric field of its own. This is a much more difficult task than in the case of the body. There is, however, some empirical evidence for an electrical or energetic aspect of the essence: three NDErs in Moody's sample referred to the essence as (1) composed of "waves" and being "charged" (Moody, 1975, p. 48); (2) being an "energy pattern" (Moody, 1975, p. 49); or (3) "a little ball of energy" (Moody, 1975, p. 50). In addition, Moody reported that almost everyone in his sample described the essence as having extension in space and as being composed of some sort of substance (Moody, 1975). There is reason, then, to proceed with the notion that the essence 
is associated with a spatially extended electric field, and to explore the ramifications of that notion.

First, let us review some of the properties of electric fields in order to simplify the discussion. The electric field generated by an arbitrarily shaped collection of electric charges (an arbitrary charge distribution) can be represented mathematically by a summation of an infinite number of algebraic terms (Lorrain and Corson, 1970). For a distribution with no net charge, the first term of this infinite series represents the field produced by an electric dipole, which consists of two charges of equal magnitude and opposite signs, separated by a fixed distance. The dipole is the simplest possible distribution of charge. The second term of the infinite series represents an electric quadrupole, and each following term grows progressively more complex. The first term, however, dominates the series, especially as distance from the distribution increases. One can therefore use the dipole as a conceptual tool in the reasoning that follows.

To a first approximation, the electric fields of the body and of the essence can be represented as dipoles. One can then ask the question, how do dipoles interact? Dipole/dipole interaction is attractive or repulsive, depending on the relative orientations of the two dipoles. The interaction is maximally attractive when the dipoles are in the antiparallel relationship (Jackson, 1975), to be discussed below. Thus a dipole/dipole attraction occurs when two dipoles are in the proper relative orientations. The force of attraction is inversely proportional to the fourth power of the distance between the dipoles.

An analogy with magnetism is illuminating here. Permanent bar magnets (magnetic dipoles) possess two ends or poles arbitrarily designated as north (N) and south (S). Similar poles held in close proximity repel each other, while opposite poles attract each other. If two magnetic dipoles are oriented so that two N-S pairs are formed (the antiparallel configuration), the magnets are found to attract each other strongly. When one varies the distance between antiparallel magnets, one can feel the rapid change in the attractive force.

In a thought experiment, one may imagine that one magnet can be switched on and off; this magnet then represents the body. The two magnets are separated by a short distance and the body magnet is switched on. The two magnets immediately and forcefully unite. Such an event could easily be described as falling like "dead weight" (Case 1) or as "two forces coming together in a crash" (Case 2).

If such a powerful electromagnetic force is at work between body and essence, then what is the significance of the essence's failure to 
interact physically with bodies other than its own (Cases 4-6)? There are at least two reasonable hypotheses, not mutually exclusive, concerning this question. The first involves concepts already encountered. When two physical dipoles closely approach each other due to mutual attraction in the antiparallel configuration, they form a quadrupole, which generates a field strength considerably less than that of a dipole. Because both dipoles are physical, that is, composed of mass, their motion towards each other is halted by the exclusionary nature of matter.

But if one dipole were not material, then the two approaching dipoles would be free to occupy the same space. As a result of this superposition, the dipolar fields would cancel each other, and the composite entity would exert no attractive (or repulsive) electromagnetic force. On this basis, one would expect that an essence could not interact with a composite of body and essence.

A second hypothesis concerning noninteraction involves the idea of tuning: perhaps the essence is specifically tuned to the NDEr's body, much the same as a radio can be tuned to a given frequency. Such a proposal would require that each body generate a characteristic field, an electromagnetic fingerprint, to which the essence could adjust. An example of this type of field is provided by an oscillating dipole with a characteristic frequency of oscillation (Lorrain and Corson, 1970). The physics of tuning is, in fact, familiar in everyday experience. Radio and television waves (and other forms of radiation) commonly pass through the body without interacting with it; humans are totally oblivious to the presence of these waves. And yet the waves interact strongly with properly tuned detection systems, allowing the reception of information.

\section{Essence /Brain Interaction}

Having addressed the question of essence/body interaction on a macroscopic scale, the theory-building process next turns to the problem of interaction of a more intimate type. Cases 7-9 demonstrate that essence and brain do interact, apparently to the detriment of essential cognitive processes. Is this finding consistent with the electromagnetic model?

Once again, the concept of dipoles is pertinent to this question. In the context of essence/brain interaction, however, the dipole is no longer meant to represent the entire entity (body or essence) but 


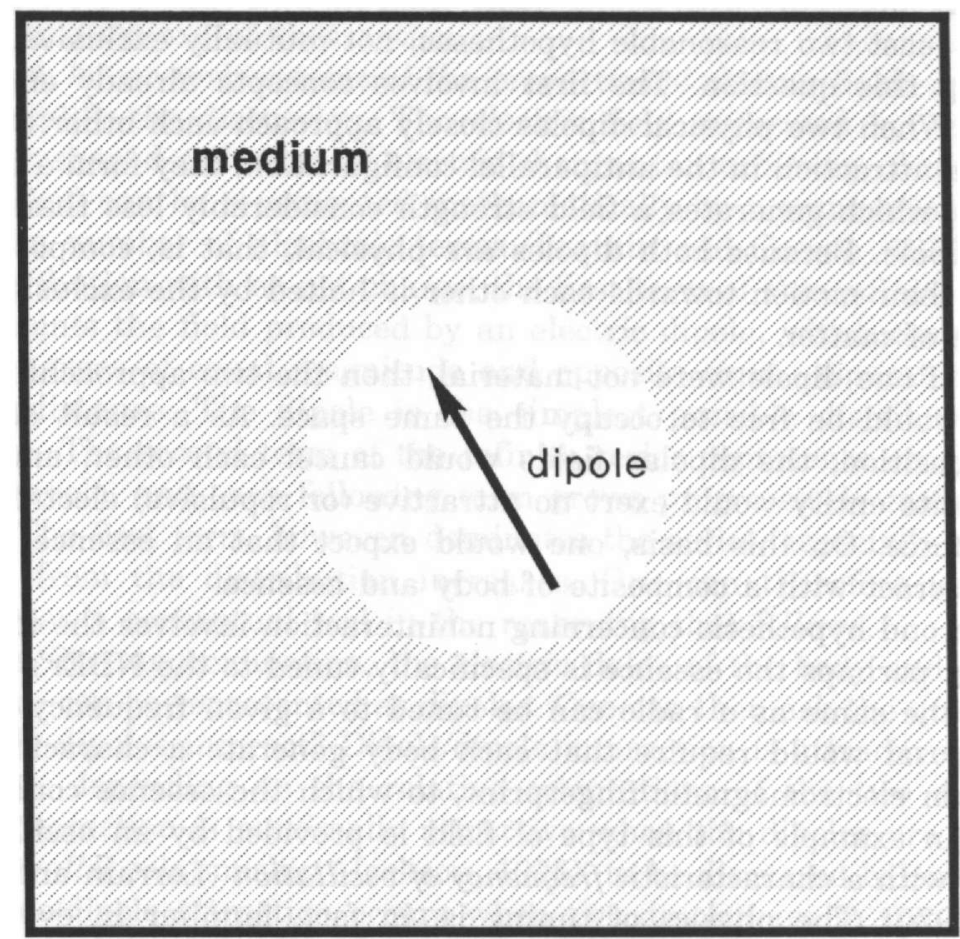

Figure 1. A cavity model for rotational relaxation. The spherical cavity is occupied by a single dipole at the cavity's center. The negatively charged end of the dipole is denoted by the arrowhead. The surrounding medium is considered to be continuous.

instead to model changes in the electric field on a microscopic scale. In this regard, concepts from an area of theoretical chemical physics called dipolar rotational relaxation (Arnette, 1981) are especially useful.

Consider a very simple model of interactions between molecules in a gas or liquid, illustrated in Figure 1. One imagines a single, rodlike, dipolar molecule, fixed in the center of an otherwise vacant spherical cavity carved out of the gaseous or liquid medium. The medium outside the cavity consists of dipolar molecules as well, but for simplicity the medium is assumed to be continuous. Both the dipole and the medium generate electric fields; the field of each is al- 
lowed to exert effects upon the other. The dipole within the cavity is free to rotate about the center of the cavity in response to changes in the medium's field, and the medium is free to respond to the motion of the dipole. These are the only interactions allowed; that is, no collisions or other rearrangements occur.

It is a basic fact of electrodynamics that a dipole will align itself in the direction of an electric field imposed upon the dipole (Lorrain and Corson, 1970); this is analogous to the northward orientation of a compass needle in the Earth's magnetic field. In the present model, the medium imposes a field on the dipole, which tends to align itself with this field. But the dipole has a field of its own, which influences the orientations, and thus the electric fields, of the dipoles in the surrounding medium.

If one could freeze all molecular motion in the model system and investigate the fields both inside and outside the cavity, one would find that the dipole has polarized the medium; that is, the electric field of the medium is distorted from its configuration in the absence of the dipole (Jackson, 1975). The field resulting from this polarized medium is called the reaction field (Onsager, 1936), since the polarization is a reaction of the medium to the dipole.

In real systems, all the molecules are in constant motion due to thermal energy. A reaction field exists at any one instant, but at the next moment the dipole has reoriented. The medium must rearrange in response to the dipole's new orientation; at the same time, the motion of the dipole is slowed by interaction with the previously formed reaction field. This last effect is termed the lag of the reaction field. It occurs because the medium requires time to respond to the dipole's motion; the reaction field thus lags behind the dipole. Since the dipole tends to align with the imposed (reaction) field, the dipole experiences a drag or dielectric friction on its reorientation.

The model for rotational relaxation just described is illustrative of a purely electromagnetic interaction. The dipole and the medium interact through a mediating electric field, which is a manifestation of both the dipole and the medium. Dipole and medium are intimately intertwined; changes in the state of one cause immediate changes in the state of the other, via the mediating field. One could say that the field embodies the interaction between dipole and medium.

Essence/brain interactions can be understood within the context of the rotational relaxation model, but only after a proposition is made about the nature of essential cognitive processes. That proposition is that the dynamic thought process of the essence is associated with 
a temporally varying and spatially extended electric field. That is, as the essence thinks, the field associated with the essence fluctuates. This is the case in the living human being, as demonstrated by electroencephalographic techniques (Carlson, 1986). Transferring this characteristic to the essence allows a description of essence/brain interactions in physical terms.

Suppose that the dipole in the rotational relaxation model represents the essence on a microscopic level, and is the driving force for cognition-that is, that the essence is the seat of thought. In light of the above proposition, the essential thought process then corresponds to the motion of the dipole. As has been seen above, dipolar motion affects the state of the medium, which in this analogy represents the brain. Thus, in the union between brain and essence, thought is integrally linked to the brain and is carried out in the presence of the brain's electric field. Two things are accomplished through this linkage: (1) sensory and other information from the body is available to the essence via the brain's electric field, and (2) the essence can influence the body by interacting with the brain's electric field.

The field representation of brain/essence union has another ramification: interaction of the brain field with the essence field interferes with or hinders the essential cognitive process. This is represented clearly in the rotational relaxation model as friction on the dipole due to the lag of the reaction field. This effect is demonstrated empirically in Cases 7-9.

\section{Essence/Essence Interaction}

The remaining question is that of the interaction of the essence with other essences. The data indicate that the essence can send and receive information at a distance. One $\mathrm{NDEr}$ characterized this process as a voice "being projected into my mind" (Case 12). Such an event is unknown in ordinary experience and in the classical physics of the macroscopic world. What is unknown in the classical world, however, can be quite common in the world of molecules.

Some molecules may, when exposed to light of the proper wavelength, absorb a photon of that light, and thus make the transition from the ground state to the excited state. The excited state has a brief lifetime, then the energy stored in the molecule finds an outlet. In ordinary fluorescence, part of the energy is re-emitted as a photon of light, and the molecule returns to the ground state (Lakowicz, 
1983). In the phenomenon known as fluorescence energy transfer (Cantor and Schimmel, 1980; Fairclough and Cantor, 1978), the energy is transferred from the original molecule (the donor) to another nearby molecule (the acceptor). This transfer is accomplished without a photon being generated. If one were able to observe the donor/acceptor system without interfering with it, one would see the excited donor and ground state acceptor, and a brief moment later the ground state donor and excited acceptor, with no observable transfer of the energy.

Such behavior can be understood on the basis of quantum mechanics, the physics of molecules' interactions with each other and with energy. The excited donor and ground state acceptor are dipoles. As such, they can interact with each other at a distance, as was shown above. In dipole/dipole coupling, a potential energy of interaction exists between the two dipoles and depends on their relative orientations. Quantum mechanics allows the transfer of energy between two coupled dipoles. The acceptor acts as a sort of antenna, receiving a signal from the excited donor. The probability of the transfer occurring depends on the nature of the medium between donor and acceptor, the dipolar orientations, and on the distance between the molecules: the greater the distance, the lower the probability. This last aspect of energy transfer has made it a useful tool for the measurement of molecular distances (Arnette, Reedquist, Barisas, and Roess, 1990; Stryer, 1978).

If indeed electromagnetic energy is a manifestation of the essential thought process, as proposed above, then the experiences reported in Cases 10-12 can be seen as analogous to fluorescence energy transfer. Information (energy) is transferred between essences (molecules) at a distance with no apparent medium or mechanism of transfer. Information can in fact take the form of energy. The energy stored in the excited molecule is information in its simplest form, similar to a computer bit switched from off to on. In the case of essence interactions, the process is, of course, much more complex than in this simple analogy; but the important point is that information exchange has a precedent in the properties and behavior of dipoles.

\section{Discussion}

The electromagnetic-quantum mechanical perspective is very useful in organizing the NDE data. Interactions of the essence with bod- 


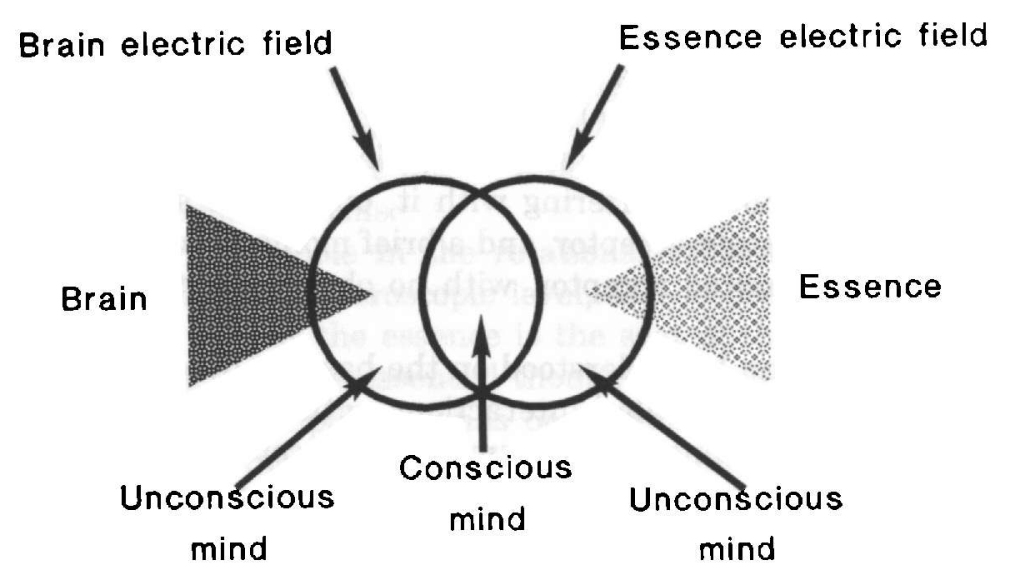

Figure 2. A structural model for a definition of mind. Conscious mind is defined as the overlap of the two fields, while unconscious mind is defined as the portions of the two fields that do not overlap.

ies, the brain, and other essences lend themselves to analogies with dipole/dipole attraction, dipolar rotational relaxation, and fluorescence energy transfer, respectively. The actual physics at work in essence interactions is undoubtedly more intricate than that of the dipole model described; the power of the dipole model lies in the fact that essence and dipole display similar types or classes of behavior. These classes are not independent of each other, but rather are linked by the physics behind the phenomena. Thus, the essence is not beyond understanding, but can be described by a set of scientific laws that have analogues in the physics of the natural world. The building of the theory of essence provides a scientific framework for the essence, and allows empirically testable predictions from that framework. One such prediction will be presented below.

\section{A Definition of Mind}

The conscious mind is that entity that is thinking, aware, and rational. It is capable of introspection, insight, and reflection. It is also in touch with sensory, physiological, and other kinds of information associated with the body. The mind can sense and process this in- 
formation and is thus affected by it; the mind can also initiate physical action based on bodily data or on the results of introspection. The mind is in this position of control by virtue of its access to both the physical and essential worlds. Mind is the interface between these worlds.

Figure 2 is a diagrammatic representation of mind based upon the rotational relaxation model of essence/brain interaction. The two Cartesian substances, essence and body, are represented by triangles. Their associated electric fields take the form of circles. The conscious mind is symbolized as the overlap of the two fields, in keeping with the rotational relaxation concept. Thus, the conscious mind is a composite, possessing elements of both body and essence. It allows causal linkage between the two substances. The electromagnetic model for interactionism was foreseen by John Wisdom (1952), who suggested that this branch of physics could provide the basis of interaction for two otherwise disparate entities.

The representation in Figure 2 also allows for mind outside of consciousness. The unconscious mind is manifested by those portions of the two fields that do not directly overlap. The conscious mind may have access to information in those portions of the fields, but that information is by definition not directly in consciousness, and its origin may be a mystery to the conscious mind. In this model, the unconscious mind is a composite in a different sense from the dualistic conscious mind.

\section{Comparison with Descartes's Propositions}

Body and essence are different substances in the Cartesian sense. Essence does not fit the definition of matter, since it does not exclude matter-as seen above-and is unaffected by gravity (Arnette, 1992). Body is matter, and is extended in all of Descartes's senses of that word. Whether body without essence is completely nonthinking is uncertain; what is empirically clear from Cases 7-9 is that essence is indeed a thinking substance. Essence is extended in the sense that it is not dimensionless or pointlike and can interface with the threedimensional brain. Essence is unextended in the sense that it does not exclude matter from its space. Essence is also indivisible, in the physical sense of that word.

Body and essence form a union, defined above as mind, that allows each substance to influence the other. The union is possible only be- 
cause body and essence are posited to have a common characteristic: both are associated with temporally varying, spatially extended electric fields. The mind consists of the overlap of these fields, and thus is different from either field alone and incomplete without both contributions.

Descartes's definition of mind and the present concept of essence have many similarities, as pointed out above, but there are also important differences. The largest points of departure between the two conceptions are the notion of extension and the nature of the characteristics common to both mind (or essence) and body. The Cartesian mind is unextended, and since Descartes did not differentiate among the various aspects of that term, he implied that the mind was dimensionless. The theory of essence explicitly holds that the essence is indeed spatially extended, but, like Descartes's concept of mind, does not exclude matter. Descartes's proposed common traits did not provide a basis for interaction. The theory of essence specifies that the common trait of an associated electromagnetic field allows for interaction.

In the development of the theory of essence, it was necessary to propose that body and essence were not completely different. If one requires of dualism that body and essence must be completely different, a radical dualist view, then the present theory is not dualistic. Interactionist theories, by their very nature, however, must require some body/essence commonality and are therefore not radically dualistic. Essence must have some aspect that we would call physical in order for essence/body interaction to occur; the body must possess something essential as well. Essence and body are extremely different, and in this sense the term dualism is appropriate. But the two must in some way speak a common language in order to communicate.

\section{A Possible Experimental Test of the Theory}

Because the essence is posited to have some physical characteristics, an experimental test of the theory is conceivable. One may be able to exploit the proposed electromagnetic nature of the essence in order to detect its presence outside the body. During the autoscopic phase of the NDE, experiencers consistently report that their consciousness is located above the body near the ceiling of the room and at a diagonal angle to the body (Moody, 1975; Ring, 1980; Sabom, 
1982). It is possible that the essence can be detected in such a situation, for example, by antennas connected with frequency analyzers.

Consider the following scenario. An operating room is specially equipped to detect and localize the presence of electromagnetic fields. The equipment is activated and monitored on a regular basis. $\mathrm{Pa}$ tients are not notified of the measurements, but are interviewed following surgery to identify cases of the NDE. Such occurrences are then correlated with the electromagnetic measurements.

If the theory of essence is correct, then the potential for success of this test rests upon the degree to which the electromagnetic properties of the essence can be detected physically. Two of the key practical problems involved are the selections of the frequency and intensity of the field to be detected. Perhaps some insight into these problems would be provided by investigations of the fields generated by living bodies. One must remember, however, that the essence may have unknown properties that would preclude physical measurements. Thus, negative results would not necessarily disprove the theory. Positive results, on the other hand, would provide powerful evidence.

\section{Conclusion}

According to the theory of essence, the body acts as a transformational system for the essence. The body detects electromagnetic, auditory, thermal, chemical, and mechanical information and transforms it via the peripheral and central nervous systems into an electromagnetic energy pattern in the brain that the essence can sample. The essence is dependent on the accuracy and integrity of physical (biological) systems, both sensory and motor, for the sensory data and physical causality it needs in order to negotiate the world and life in it.

This theory generates a set of questions centering on the biological details and mechanisms of essence/brain interaction, which fall into two general categories: anatomical and physiological. We might ask, for example, about details of the essence/brain interface, where in the brain this interface might occur, what supporting evidence there is for any such localization, and the physiological mechanisms of interaction on the molecular level in the interface. These aspects of the theory are currently under development. 


\section{References}

Arnette, J. K. (1981). Rotational relaxation of a dipole in two dimensions. Unpublished doctoral dissertation, Florida State University, Tallahassee, FL.

Arnette, J. K. (1992). On the mind/body problem: The theory of essence. Journal of Near-Death Studies, 11, 5-18.

Arnette, J. K., Reedquist, K., Barisas, B., and Roess, D. (1990). FET measurement of interprotein distances in membrane protein aggregates on single cells. Biophysical Journal, 57, 252a.

Becker, H., and Geer, B. (1982). Participant observation: The analysis of qualitative field data. In Burgess, R. (Ed.), Field research: A sourcebook and field manual. London: George Allen and Unwin.

Blumer, H. (1969). Symbolic interactionism. Englewood Cliffs, NJ: Prentice-Hall.

Cantor, C., and Schimmel, P. (1980). Biophysical chemistry II: Techniques for the study of biological structure and function. San Francisco, CA: Freeman.

Carlson, N. (1986). Physiology of behavior. Boston, MA: Allyn and Bacon.

Dennett, D. (1991). Consciousness explained. Boston, MA: Little, Brown.

Descartes, R. (1986). Meditations on first philosophy (J. Cottingham, Trans.). Cambridge, England: Cambridge University Press. (Original work published 1641)

Einstein, A. (1961). Relativity. New York, NY: Crown.

Fairclough, R., and Cantor, C. (1978). The use of singlet-singlet energy transfer to study macromolecular assemblies. Methods in Enzymology, 48, 347-379.

Holsti, O. (1969). Content analysis for the social sciences and humanities. Reading, MA: Addison-Wesley.

Jackson, J. (1975). Classical electrodynamics. New York, NY: Wiley.

Kuhn, T. (1970). The structure of scientific revolutions. Chicago, IL: University of Chicago Press.

Lakowicz, J. (1983). Principles of fluorescence spectroscopy. New York, NY: Plenum Press.

Lange, F. (1925). The history of materialism (E. Thomas, Trans.). London: Routledge and Kegan Paul. (Original work published 1865)

Loeb, L. (1981). From Descartes to Hume. Ithaca, NY: Cornell University Press.

Lorrain, P., and Corson, D. (1970). Electromagnetic fields and waves. San Francisco, CA: Freeman.

Misner, C., Thorne, K., and Wheeler, J. (1973). Gravitation. San Francisco, CA: Freeman.

Moody, R. (1975). Life after life. Covington, GA: Mockingbird Books.

Onsager, L. (1936). Electric moments of molecules in liquids. Journal of the American Chemical Society, 58, 1486-1493.

Popper, K., and Eccles, J. (1977). The self and its brain. New York, NY: Springer-Verlag.

Radner, D. (1971). Descartes's notion of the union of mind and body. Journal of the History of Philosophy, 9, 159-170.

Radner, D. (1985). Is there a problem of Cartesian interaction? Journal of the History of Philosophy, 23, 35-49.

Ring, K. (1980). Life at death: A scientific investigation of the near-death experience. New York, NY: Coward, McCann and Geoghegan.

Ring, K. (1984). Heading toward omega: In search of the meaning of the near-death experience. New York, NY: Morrow.

Sabom, M. (1982). Recollections of death: A medical investigation. New York, NY: Harper and Row.

Sarasohn, L. (1985). Motion and morality: Pierre Gassendi, Thomas Hobbes, and the mechanical world-view. Journal of the History of Ideas, 46, 363-379. 
Stryer, L. (1978). Fluorescence energy transfer as a spectroscopic ruler. Annual Review of Biochemistry, 47, 819-846.

Tesch, R. (1990). Qualitative research: Analysis types and software tools. Bristol, PA: Burgess Science Press.

Wisdom, J. (1952). A new model for the mind-body relationship. British Journal for the Philosophy of Science, 2, 295-301. 